\title{
Beiträge zur Kenntnis der in ungekeimten Pflanzensamen enthaltenen Stickstoffverbindungen.
}

Von

E. Schulze und N. Castoro.

(Aus dem agrikultur-chemischen Laboratorium des Polytechnikums in Zürich.)

(Der Redaktion zugegangen am 31. März 1904.)

Bekanntlich enthalten die Pflanzensamen neben Proteinstoffen fast immer nichtproteinartige Stickstoffverbindungen in kleiner Menge. Wie viel Stickstoff auf die zuletzt genannten Verbindungen fällt, hat man mit Hilfe der Stutzerschen Methode zu bestimmen gesucht. Die dabei erhaltenen Resultate, welche für eine ansehnliche Zahl von Pflanzensamen vorliegen, zeigen bedeutende Schwankungen. Sehr niedrig sind sie bei Koniferensamen, indem hier die auf nichtproteinartige Verbindungen fallende Stickstoffmenge nur $0,03-0,095 \%$ der Samentrockensubstanz betrug. ${ }^{1}$ ) Für die Samen landwirtschaftlicher Kulturpflanzen werden folgende Durchschnittszahlen angegeben: ${ }^{2}$ )

\begin{tabular}{|c|c|c|c|c|c|}
\hline Weizen & $0,240 \%$ & $=$ & $11,2 \%$ & les & stickstoffs \\
\hline Roggen & $0,195 \%$ & $=$ & $9,4 \%$ & $\gg$ & $>$ \\
\hline Gerste & $0,051 \%$ & $=$ & $2,6 \%$ & > & s \\
\hline Hafer & $0,125 \%$ & $=$ & $7,5 \%$ & $D$ & $\triangleright$ \\
\hline Mais & $0,090 \%$ & $=$ & $4,9 \%$ & $>$ & > \\
\hline Erbse & $0,543 \%$ & $=$ & $11,4 \%$ & $>$ & > \\
\hline Ackerbohnen & $0,562 \%$ & $=$ & $11,4 \%$ & $\gg$ & $>$ \\
\hline Sojabohne & $0,668 \%$ & $=$ & $10,1 \%$ & 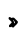 & $>$ \\
\hline Lupine, gelbe & $0,628 \%$ & $=$ & $8,8 \%$ & > & $>$ \\
\hline$\gg \quad$ blaue & $0,410 \%$ & $=$ & $7,7 \%$ & 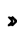 & 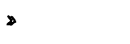 \\
\hline Leinsamen & $0,200 \%$ & $=$ & $5,5 \%$ & 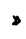 & > \\
\hline
\end{tabular}

1) E. Schulze, Über die Zusammensetzung einiger Koniferensamen, Landw. Versuchsstationen, Bd. 55, S. 277.

2) Wolff-Lehmann, Landw. Fütterungslehre, S. 248. 
Den in unserem Laboratorium nach dem gleichen Verfahren ausgeführten Bestimmungen entnehmen wir noch folgende Zahlen:

$\begin{array}{ll}\text { Wicke } & 0,504 \%=10,0 \% \text { des Gesamtstickstoffs } \\ \text { Weiße Lupine, entschält } \begin{array}{l}0,790 \%=10,3 \% \\ \text { Sonnenblume }\end{array} & 0,100 \%=2,5 \% \\ \text { Hanf } & 0,390 \%=10,3 \% \\ \text { Kürbis, entschält } & 0,150 \%=3,4 \%\end{array}$

Wie man aus vorstehenden Angaben ersehen kann, liegen die in solcher Weise gefundenen Zahlen bei ölreichen Samen und bei Getreidekörnern niedriger, als bei Leguminosen-Samen. Bei verschiedenen Mustern der gleichen Samenart zeigten sich aber bedeutende Schwankungen; für die Samen der gelben Lupine z. B. werden Schwankungen von $0,41-1,22 \%,{ }^{1}$ ) für Weizenkörner solche von $0,18-0,30 \%$ angegeben. Allem Anschein nach ist es von Einfluß, ob die Samen besser oder weniger gut ausgereift sind; denn in unreifen Samen hat man in der Regel mehr nichtproteinartige Stickstoffverbindungen gefunden, als in reifen.

Auf die Frage nach der Natur der Stickstoffverbindungen, die sich in den nach Stutyers Vorschrift zur Ausfällung der Proteinstoffe mit Kupferhydroxyd erhitzten Extrakten aus Pflanzensamen noch in Lösung vorfinden, läßt sich eine erschöpfende Antwort zur Zeit nicht geben. Man weiß allerdings, daß manche der obengenannten Samenarten stickstofthaltige Basen, wie Cholin, Betain, Trigonellin usw., enthalten ${ }^{2}$ ) und daß neben letzteren hin und wieder auch stickstoffhaltige Glukoside, wie Amygdalin und Vicin, vorkommen; doch ist, so viel man wciß, dor Gchalt daran so gering, daß in den meisten Fällen durch diese Stoffe ohne Zweifel nur ein Teil

1) Für eines der in unserem Laboratorium untersuchten Muster der Samen von Lupinus luteus wurde eine Zahl erhalten, die noch unter dem oben als Minimum angegebenen Wert liegt, nämlich $0,24 \%$ (für entschälte Samen).

2) M. vgl. die Abhandlung E. Schulzes «Untersuchungen über die zur Klasse der stickstoffhaltigen organischen Basen gehörenden Bestandteile einiger landwirtschaftlich benutzten Samen, Ölkuchen und Wurzelknollen, sowie einiger Keimpflanzen». Landwirtschaftl. Versuchsstationen, Bd. 44 , S. 23-77. 
der Stickstoffmenge gedeckt wird, die nach den in der angegebenen Weise ausgeführten Bestimmungen auf nichtproteinartige Verbindungen fällt.

Bei Ausführung der Untersuchungen über den Eiweißumsatz in Keimpflanzen, deren Ergebnisse von E. Schulze und seinen Mitarbeitern in einer Reihe von Abhandlungen mitgeteilt worden sind, war es von Interesse, zu prüfen, ob schon in den ungekeimten Samen Asparagin oder Aminosäuren oder andere Produkte des Eiweißumsatzes sich vorfinden. Daß die Quantität solcher Produkte in den ungekeimten Samen nur eine sehr geringe sein konnte, war aus dem niedrigen Stickstoffgehalt der von den Eiweißstoffen so vollständig wie möglich befreiten wässerigen Extrakte zu schließen. Auch erhielt man bei dem Versuche, aus ungekeimten Samen Asparagin oder Aminosäuren $\mathrm{zu}$ isolieren, fast ausnahmslos negative Resultate; nur aus dem Embryo des Weizenkorns konnte eine sehr kleine Quantität von Asparagin abgeschieden werden. Doch können, wie in den bezüglichen Mitteilungen hervorgehoben ist, die negativen Resultate nicht als ein sicherer Beweis für das völlige Fehlen der genannten Stickstoffverbindungen in den Untersuchungsobjekten angesehen werden, weil die in Anwendung gebrachten Verfahren die Auffindung sehr kleiner Quantitäten jener Substanzen kaum gestatten.

Bei Ausführung von Versuchen über die Bildung von Arginin bei der Autolyse (Autodigestion) von Lupinus-Keimpflanzen untersuchten wir auch ungekeimte Lupinussamen auf Arginin; dabei stellte sich heraus, daß Samen von Lupinus luteus schon vor der Keimung eine nicht ganz unbeträchtliche Quantität der genannten Base enthielten. Infolge davon haben wir auch noch einige andere Samenarten auf Arginin untersucht und diese Objekte zugleich auf das Vorhandensein anderer nichtproteinartiger Stickstoffverbindungen geprüft. Die dabei erhaltenen Resultate teilen wir im folgenden mit.

1. Samen der gelben Lupine (Lupinus luteus).

Zur Untersuchung gelangten drei Muster solcher Samen, von denen eines aus Frankreich, die beiden anderen aus 
Deutschland bezogen worden waren. Die Darstellung von Arginin aus diesen Objekten geschah in folgender Weise: Die gepulverten Samen wurden in Wasser, welches eine Temperatur von ungefähr $90^{\circ}$ besaß, unter Umrühren nach und nach eingetragen. Nach Verlauf von einigen Stunden trennten wir den wässerigen Auszug vom ungelösten Rückstand und versetzten ihn mit Tannin, bis dieses Reagens nur noch eine ganz schwache Fällung hervorbrachte. Der durch Filtration vom Niederschlage getrennten Flüssigkeit fügten wir Bleiessig in schwachem Überschuß zu. Das Filtrat vom Bleiniederschlage wurde bei ganz schwachsaurer Reaktion im Wasserbade eingeengt, hierauf mit Schwefelsäure stark angesäuert und nach nochmaliger Filtration mit Phosphorwolframsäure versetzt, so lange als dieses Reagens noch eine sofort entstehende Fällung hervorbrachte. Den Phosphorwolframsäureniederschlag zerlegten wir, nachdem er abfiltriert und mit $5 \%$ iger Schwefelsäure ausgewaschen worden war, in bekannter Weise durch Baryumhydroxyd. Aus der dabei erhaltenen Basenlösung fällten wir nach dem Verfahren von Kossel und Kutscher durch Silbernitrat und Barytwasser zuerst das Histidin, dann das Arginin aus. Der Argininsilberniederschlag wurde nach der von den genannten Forschern gegebenen Vorschrift behandelt, das dabei erhaltene Arginin in das Nitrat übergeführt, die wässerige Lösung des letzteren im Wasserbade zur Sirupskonsistenz eingedunstet. Der Sirup verwandelte sich bald nach dem Erkalten in eine fast farblose Kristallmasse, in welcher das Vorhandensein von Mutterlauge nicht mehr zu bemerken war. Diese Kristallmasse war fast frei von Asche und bestand ohne Zweifel aus fast völlig reinem Argininnitrat. Das daraus dargestellte Argininkupfernitrat kristallisierte sofort in der charakteristischen Form, nämlich in dunkelblauen, halbkugelförmigen, aus feinen Prismen zusammengesetzten Aggregaten. Dieses Produkt schmolz, ohne zuvor umkristallisiert worden zu sein, bei $113-114^{\circ}$. Eine Probe desselben wurde mit Hilfe von Schwefelwasserstoff vom Kupfer befreit; das so erhaltene neutrale Nitrat gab die Argininreaktionen. ${ }^{1}$ )

1) M. vgl. in Betreff dieser Reaktionen: Diese Zeitschr., Bd. XI, S. 56. 
Wenn auch die im vorigen mitgeteilten Versuchsergebnisse es schon zweifellos machten, daß Arginin vorlag, so haben wir doch auch noch Kupferbestimmungen im Argininkupfernitrat ausgeführt. Dabei erhielten wir für die zuvor durch vorsichtiges Trocknen (zuerst bei 80 , dann bei $100^{\circ}$ ) vom Kristallwasser befreite Substanz folgende Resultate:

1. $0,3375 \mathrm{~g}$ Substanz gaben $0,0500 \mathrm{~g} \mathrm{CuO}=11,83 \% \mathrm{Cu}$

2. 0,3375 , " 0,0495 » $>11,72 \%$,

Die Theorie verlangt $11,89 \% \mathrm{Cu}$.

Die Ausbeute an Arginin zeigte bei den zur Untersuchung gelangten drei Samenmustern keine großen Differenzen, wie aus nachfolgenden Angaben $\mathrm{zu}$ ersehen ist:

Samen A. $194,8 \mathrm{~g}$ der Samentrockensubstanz lieferten $1,113 \mathrm{~g}$ Argininnitrat $=0,7902 \mathrm{~g}$ oder 0,41\% Arginin.

Samen B. $199,1 \mathrm{~g}$ der Samentrockensubstanz lieferten $0,838 \mathrm{~g}$ Argininnitrat $=0,595 \mathrm{~g}$ oder $0,30 \%$ Arginin.

Samen C. $150,4 \mathrm{~g}$ der Samentrockensubstanz lieferten $0,795 \mathrm{~g}$ Argininnitrat $=0,564 \mathrm{~g}$ oder 0,38\% Arginin.

Im Durchschnitt haben also 100 Teile der Samentrockensubstanz 0,36 Teile Arginin geliefert; die auf diese Base fallende Stickstoffmenge würde demnach $0,115 \%$ der Samentrockensubstanz betragen haben. Doch ist anzunehmen, daß diese Zahlen etwas zu niedrig gefunden wurden; denn abgesehen davon, daß die Ausfällung des Arginins aus den Extrakten keine ganz vollständige war, weil das Phosphorwolframat des Arginins in kaltem Wasser nicht ganz unlöslich ist, liegt es auch im Bereich der Möglichkeit, daß die Operationen, denen die Extrakte vor der Ausfällung des Arginins unterworfen wurden, kleine Verluste an jener Base verursachten, indem vielleicht in den durch Tannin und durch Bleiessig erzeugten starken Niederschlägen kleine Argininmengen zurückgeblieben sind.

Wahrscheinlich wurde das Arginin von einer kleinen Menge von Histidin begleitet; wenigstens fand sich eine Substanz vor, welche wie das Histidin nicht nur durch Silbernitrat und Barytwasser, sondern auch durch ammoniakalische Silberlösung und durch Quecksilbersulfat gefällt wurde. Doch war ihre Quantität zu gering, um sie rein darzustellen und mit Sicherheit zu identifizieren.

Hoppe-Seyler's Zeitschrift f. physiol. Chemie. XLI. 
Um die ungekeimten Samen auf Asparagin und auf Tyrosin zu prüfen, verfuhren wir in folgender Weise: aus einem wässerigen Extrakt aus $500 \mathrm{~g}$ der zerkleinerten Samen fällten wir die Proteinstoffe und die Basen durch Phosphorwolframsäure aus. Die durch Filtration vom Niederschlag getrennte Flüssigkeit wurde mit Bleiessig, das Filtrat vom Bleiniederschlag sodann mit Mercurinitratsolution versetzt. Das letztere Reagens brachte nur eine sehr schwache Fällung hervor, die jedoch stärker wurde, nachdem durch Zusatz von Natriumkarbonat die Acidität der Flüssigkeit abgestumpft worden war. Diese Fällung wurde abfiltriert, ausgewaschen und sodann durch Schwefelwasserstoff zerlegt. Die vom Schwefelquecksilber abfiltrierte Flüssigkeit neutralisierten wir mit Ammoniakund dunsteten sie sodann zum Sirup ein. Der letztere lieferte nach einiger Zeit eine Ausscheidung, welche umkristallisiert wurde. Dabei wurde in sehr kleiner Quantität eine in kaltem Wasser sehr schwer lösliche, in verdünnter Salpetersäure leicht lösliche Substanz erhalten, die mit Millonschem Reagens Tyrosinreaktion gab. Beim Erhitzen mit dem Mörnerschen Reagens gab sie jedoch keine rein grüne Färbung, woran vielleicht irgend eine Beimengung die Schuld trug. So können wir es denn nicht für sicher, sondern nur für wahrscheinlich erklären, daß hier Tyrosin in sehr kleiner Menge vorlag. Daneben wurden einige Kristalle erhalten, welche Asparagin zu sein schienen; doch war die Quantität derselben zu gering, um ihre Natur bestimmt erkennen zu können.

Während wir Tyrosin und Asparagin nicht sicher nachzuweisen vermochten, gelang dagegen die Isolierung einer Substanz, deren Identität mit Vernin mit Sicherheit festgestellt werden konnte. Zur Darstellung dieses Produkts diente ein wässeriges Extrakt aus 2 Kilo der zerkleinerten Samen. Wir versetzten dieses Extrakt zunächst mit Phosphorwolframsäure; doch wurde dieses Reagens nur in solcher Quantität zugefügt, daß nur ein Teil der durch dasselbe fällbaren Extraktbestandteile niedergeschlagen werden konnte. Die vom Phosphorwolframsäureniederschlag abfiltrierte Flüssigkeit wurde mit Bleiessig, das Filtrat vom Bleiniederschlag, sodann mit Mercurinitrat- 
solution versetzt, wobei ein ziemlich starker Niederschlag entstand, den wir nach dem Abfiltrieren und Auswaschen in Wasser verteilten und durch Schwefelwasserstoff zerlegten. Die vom Schwefelquecksilber abfiltrierte Flüssigkeit wurde mit Ammoniak neutralisiert und sodann zum Sirup eingedunstet. Dieser Sirup lieferte bald nach dem Erkalten eine amorphe, fast gallertartige Ausscheidung, die wir von der Mutterlauge trennten und sodann in kochendem Wasser aufösten. Aus der Lösung schieden sich beim Erkalten feine Kristalle aus, die noch einmal aus heißem Wasser umkristallisiert wurden. Das in dieser Weise erhaltene Produkt besaß alle Eigenschaften des Vernins. Es war sehr schwer löslich in kaltem, leicht in kochendem Wasser; die Lösung lieferte schon während des Erkaltens eine Ausscheidung von dünnen prismatischen Kristallen, die nach dem Abfiltrieren und Trocknen eine weiße, atlasglänzende Masse bildeten. Die wässerige Lösung der Kristalle gab mit Silbernitrat eine durchsichtige, gallertartige, in Ammoniakflüssigkeit lösliche Fällung, mit Phosphorwolframsäure einen gelblichen Niederschlag; auf Zusatz von Pikrinsäure schieden sich nach einiger Zeit kleine gelbe Kristalle von moosähnlichem Aussehen ab. Als unser Produkt ca. eine Stunde lang mit verdünnter Salzsäure gekocht, die Lösung sodann mit Ammoniak neutralisiert wurde, entstand ein gelblicher Niederschlag. Dieser Niederschlag bestand aus Guanin. Den Beweis dafür geben folgende Tatsachen: Die für Guanin zu erklärende Substanz gab die sog. Xanthinprobe. ${ }^{1}$ ) Sie löste sich in der Wärme leicht in verdünnter Salzsäure; aus der Lösung schied sich nach dem Erkalten in feinen Kristallnadeln ein Chlorhydrat ab, welches im Aussehen mit salzsaurem Guanin vollständig übereinstimmte und gleich dem letzteren bei längerem Kochen mit Wasser die Salzsäure verlor. Aus der verdünnten wässerigen Lösung dieses Chlorhydrats schieden sich auf Zusatz von Pikrinsäure nach kurzer. Zeit gelbe Kristalle ab, die das charakteristische Aussehen des Guanidinpikrats besaßen. Die gleiche Lösung

1) M. vgl. Hoppe-Seylers Handbuch der physiologisch- und pathologisch-chemischen Analyse, neu bearbeitet von Thierfelder, $\S 125$, S. 143. 
lieferte nach Zusatz einiger Tropfen konzentrierter Ferricyankaliumsolution gelbbraune Kristalle. Mit Metaphosphorsäure gab die Lösung des Chlorhydrats einen im Überschuß des Fällungsmittels unlöslichen Niederschlag; sie gab ferner mit ammoniakalischer Silberlösung eine in Ammoniak unlösliche Fällung. Diese Reaktionen lassen keinen Zweifel daran, daß Guanin vorlag; dadurch vervollständigt sich aber der Beweis dafür, daß die Substanz, welche beim Kochen mit Salzsäure dieses Produkt lieferte, Vernin war.

Bei Untersuchung unseres Verninpräparates ergab sich, daß dieser stickstoffreiche Körper noch einige Reaktionen gibt, die nicht allein zu seiner Erkennung benutzt werden können, sondern auch über den in ihm mit dem Guanin verbundenen Atomkomplex in gewissem Grade Aufschluß geben. Wenn man ein wenig Vernin in Schwefelsäure, die mit einer geringen Wassermenge verdünnt ist, auflöst, der Lösung etwas $\alpha$-Naphtol zufügt und sie sodann schwach erwärmt, so färbt sie sich zuerst rot, bald aber intensiv violett. Diese Reaktion ist sehr empfindlich; sie tritt schon bei Anwendung einer äußerst geringen Verninquantität ein. Ferner gibt das Vernin bei Erwärmen mit Salzsäure und Phloroglucin eine kirschrote Lösung; auch mit Resorcin und Salzsäure gibt es beim Erwärmen eine rote Flüssigkeit. Aus diesen Reaktionen kann man schließen, daß im Vernin eine Kohlenhydratgruppe enthalten ist. Zu erwähnen ist noch, daß beim Kochen des Vernins mit konzentrierter oder nur wenig mit Wasser verdünnter Salzsäure nach 5-10 Minuten die Flüssigkeit sich unter Abscheidung eines huminartigen Produkts dunkel färbt und daß dabei ein Geruch auftritt, wie er beim Verbrennen von Kohlenhydraten sich bemerkbar macht.

Es war nun zu prüfen, ob das Vernin bei der Spaltung durch Säuren eine die Fehlingsche Flüssigkeit reduzierende Zuckerart liefert. Das Entstehen einer reduzierenden Substanz ließ sich in der Tat in folgender Weise nachweisen: Wir er-

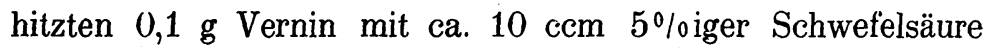
$2-2^{1 / 2}$ Stunden lang bis fast zum Sieden. Nach dem Erkalten wurde die Flüssigkeit mit Barytwasser genau neutralisiert, das 
dabei entstandene Baryumsulfat abfiltriert, das Filtrat im Wasserbade eingedunstet. Den Verdampfungsrückstand nahmen wir in Wasser auf, wobei eine kleine Substanzmenge ungelöst blieb. Die filtrierte Lösung wurde noch einmal eingedunstet, der Verdampfungsrückstand wieder in wenig Wasser gelöst. Die so erhaltene Flüssigkeit reduzierte stark die Fehlingsche Lösung. Die Ergebnisse dieser Versuche führen zu der Schlußfolgerung, daß das Vernin eine Kohlenhydratgruppe einschließt und für ein Glukosid zu erklären ist. Diese Erkenntnis macht es allem Anschein nach möglich, eine Wahl zwischen den beiden Formeln $\mathrm{C}_{10} \mathrm{H}_{13} \mathrm{~N}_{5} \mathrm{O}_{5}$ und $\mathrm{C}_{16} \mathrm{H}_{20} \mathrm{~N}_{8} \mathrm{O}_{8}$, die nach den Ergebnissen der Elementaranalysen zulässig für das Vernin sind, ${ }^{1}$ ) zu treffen. Man hat früher mit Rücksicht auf den Silbergehalt der Silberverbindung des Vernins der zuletzt aufgeführten Formel den Vorzug gegeben; doch ist jetzt wohl die erste Formel vorzuziehen. Denn wenn man diese Formel annimmt, so ist es denkbar, daß das Vernin unter Wasseraufnahme in Guanin und eine Substanz von der Zusammensetzung einer Pentose zerfällt, und zwar nach folgender Gleichung:

$$
\mathrm{C}_{10} \mathrm{H}_{13} \mathrm{~N}_{5} \mathrm{O}_{5}+\mathrm{H}_{2} \mathrm{O}=\mathrm{C}_{5} \mathrm{H}_{5} \mathrm{~N}_{5} \mathrm{O}+\mathrm{C}_{5} \mathrm{H}_{10} \mathrm{O}_{5} \text {. }
$$

Daß bei der Spaltung des Vernins eine Pentose entsteht, ist nicht unwahrscheinlich im Hinblick auf die kirschrote Färbung, die das Vernin beim Erhitzen mit Phloroglucin und Salzsäure gibt. Um diese Fragen mit Sicherheit entscheiden zu können, bedarf es neuer Versuche, die wir nach Beschaffung einer größeren Materialmenge anzustellen gedenken.

Das Vernin ist zwar in einer beträchtlichen Zahl pflanzlicher Substanzen gefunden worden, ${ }^{2}$ ) tritt aber stets nur in sehr kleiner Menge auf; die Darstellung eines größeren Quantums dieses Körpers verursacht daher viel Arbeit. Auch aus den von uns untersuchten Lupinensamen ließ sich nur eine sehr kleine Verninmenge gewinnen; in einem Versuche erhielten wir aus zwei Kilo Samen nur ungefähr ein halbes Gramm Vernin, in einem zweiten Versuche eine noch geringere Ausbeute.

1) Wir verweisen auf die Abhandlung von E. Schulze und E. Bosshard "Über das Vernin», Diese Zeitschrift, Bd. X, S. 80-89.

2) Diẹse Zeitschrift, Bd. X, S. 80 und Seite 326. 
Die Mutterlauge vom Rohvernin lieferte beim Verdunsten eine Kristallisation von Argininnitrat (bekanntlich läßt sich das Arginin aus den Pflanzenextrakten durch Mercurinitrat fällen, doch ist die Ausfällung wohl nur eine unvollständige).

In einem der für unsere Versuche verwendeten drei Samenmuster bestimmten wir die auf nichtproteinartige Verbindungen fallende Stickstoffmenge nach Stutzers Verfahren; sie betrug $0,79 \%$ der Samentrockensubstanz. ${ }^{1}$ ) Nur ein Teil dieses Betrages wird durch die von uns zur Abscheidung gebrachten Stickstoffverbindungen (Arginin und Vernin) gedeckt. Doch enthalten bekanntlich die Lupinensamen auch noch Basen, die zu den echten Alkaloiden gerechnet werden; die in Form solcher Basen in den Samen von Lupinus luteus sich vorfindende Stickstoffmenge ist aber nur auf ungefähr 0,10\% der Samentrockensubstanz zu schätzen. Demnach müssen noch andere nichteiweißartige Stickstoffverbindungen in diesen Samen enthalten sein. Vielleicht finden sich noch peptonartige Stoffe vor.

2. Samen der weißen Lupine (Lupinus albus).

Die bei Untersuchung dieser Samen auf nichtproteinartige Stickstoffverbindungen von uns erhaltenen Resultate sind schon in einer früher publizierten Abhandlung ${ }^{2}$ ) mitgeteilt worden. Wir konnten aus diesen Samen Arginin abscheiden; doch betrug die Ausbeute daran nur 0,02\% der Samentrockensubstanz. Asparagin konnten wir nicht nachweisen; in sehr kleiner Menge wurde eine Substanz erhalten, die wahrscheinlich Tyrosin war. Auch die Samen der weißen Lupine enthalten bekanntlich Basen, die zu den echten Alkaloiden gerechnet werden.

3. Samen der Sonnenblume (Helianthus annuus).

In einem von S. Frankfurt ${ }^{3}$ ) in unserem Laboratorium untersuchten Muster solcher Samen fiel 0,10\% $\mathrm{N}$ auf nichtproteinartige Verbindungen. Es war daher nicht zu erwarten, daß solche Verbindungen in beträchtlicher Quantität aus dieser

1) Analytische Belege: a) 0,900 g Trockensubstanz gaben $0,00693 \mathrm{~g}=0,77 \% \mathrm{~N}$, b) $0,900 \mathrm{~g}$ Trockensubstanz gaben $0,00724 \mathrm{~g}=$ $0,80 \% \mathrm{~N}$.

2) Diese Zeitschrift, Bd. XXXVIII, S. 221.

3) Landwirtschaftliche Versuchsstationen, Bd. 43, S. 161. 
Samenart darzustellen waren. Dieser Erwartung entsprach auch der Befund.

Die für unsere Versuche zur Verwendung gelangenden zwei Kilo Samen wurden zunächst grob zerkleinert und mit Hilfe von Äther vom größten Teil des fetten Öles befreit, dann pulverisiert und nun mit heißem Wasser extrahiert. Aus dem Extrakt suchten wir Arginin darzustellen, wobei wir so verfuhren, wie es oben bei Beschreibung der mit Lupinensamen angestellten Versuche angegeben worden ist. Wir erhielten eine sehr kleine Quantität von Argininnitrat. Dieses Salz wurde zunächst umkristallisiert, dann in Argininkupfernitrat übergeführt. Die Ausbeute an letzterem betrug etwas weniger als $0,1 \mathrm{~g}$. Da diese Verbindung aber in der gewöhnlichen Form kristallisierte, da die Kristalle zwischen 112 und $115^{\circ}$ schmolzen und bei der Zerlegung mittels Schwefelwasserstoff ein neutrales Nitrat lieferten, welches im Aussehen mit Argininnitrat übereinstimmt und die Reaktionen des Arginins gab, so kann wohl nicht bezweifelt werden, daß diese Base wirklich sich vorfand. Allerdings könnte man im Hinblick auf die geringe Ausbeute die Frage aufwerfen, ob nicht etwa das zur Ausscheidung gebrachte Arginin erst während der Verarbeitung des Extrakts sich gebildèt habe; doch glauben wir, daß diese Frage, auf die wir übrigens am Schlusse unserer Abhandlung noch einmal zurückkommen werden, zu verneinen ist.

Um noch die im Filtrat vom Argininsilberniederschlag enthaltenen Basen zu gewinnen, befreiten wir dieses Filtrat vom gelösten Silber und vom Baryt und versetzten es sodann mit Phosphorwolframsäure. Den durch dieses Reagens hervorgebrachten Niederschlag zerlegten wir durch Baryumhydroxyd und führten die in Freiheit gesetzten Basen in die salzsauren Salze über. Die wässerige Lösung der letzteren verwandelte sich, nachdem sie im Wasserbade stark eingeengt worden war, im Exsikkator bald in eine Kristallmasse. Dieselbe wurde, nachdem sie völlig trocken geworden war, mit heißem Weingeist behandelt, wobei ein fast ausschließlich aus anorganischen Chloriden bestehender Rückstand blieb. Die von diesem Rückstand getrennte weingeistige Lösung versetzten wir mit einer 
weingeistigen Mercurichloridsolution, was bald die Ausscheidung von Quecksilberdoppelsalzen zur Folge hatte. . Letztere wurden von der Flüssigkeit getrennt, unter Zusatz von etwas Mercurichlorid aus heißem Wasser umkristallisiert und sodann durch Schwefelwasserstoff zersetzt. Die vom Schwefelquecksilber abfiltrierte Flüssigkeit lieferte beim Eindunsten einen Rückstand, welcher zwei Chlorhydrate einschloß. Sie ließen sich durch Behandlung mit kaltem absoluten Alkohol trennen. $\left.{ }^{1}\right)$ Das darin lösliche Chlorhydrat erwies sich als salzsaures Cholin. Das daraus dargestellte Chloroplatinat kristallisierte wie Cholinplatinchlorid beim langsamen Verdunsten seiner wässerigen Lösung in orangeroten Tafeln, deren Platingehalt dem von der Formel des Cholinplatinchlorids geforderten Werte $(31,6 \% \mathrm{Pt})$ entsprach, wie folgende Angaben beweisen:

$0,2405 \mathrm{~g}$ über Schwefelsäure, dann bei $100^{\circ}$ getrocknete Substanz gaben $0,0755 \mathrm{~g}=31,4 \% \mathrm{Pt}$.

Das aus dem Chloroplatinat wieder dargestellte Chlorhydrat der Base kristallisierte in zerfließlichen Nadeln und gab die Reaktionen des Cholins. ${ }^{2}$ )

Das zweite Chlorhydrat, welches in kaltem absoluten Alkohol fast unlöslich war, zur Reinigung aber noch aus Wasser umkristallisiert wurde, kann für salzsaures Betain erklärt werden. Das daraus dargestellte, in kaltem Wasser schwer lösliche Chloraurat kristallisierte wie Betaingoldchlorid in glänzenden Blättchen und Nadeln und besaß einen der Theorie $(43,1 \% \mathrm{Au})$ entsprechenden Goldgehalt, wie folgende Angaben beweisen:

$0,189 \mathrm{~g}$ über Schwefelsäure, dann bei $100^{\circ}$ getrocknete Substanz gaben $0,081 \mathrm{~g}=42,86 \% \mathrm{Au}$.

Das bei Zerlegung des Chloraurats mittels Schwefelwasserstoff erhaltene Chlorhydrat kristallisierte in ziemlich großen luftbeständigen Kristallen und gab die Reaktionen des Betains. ${ }^{3}$ )

1) Doch ist die Trennung keine ganz scharfe.

2) M. vgl. in Betreff dieser Reaktionen Diese Zeitschr., Bd. XI, S. 369.

3) Als besonders wichtig kann die Reaktion mit Kaliumquecksilberjodid bezeichnet werden. Der durch letzteres hervorgebrachte Niederschlag löst sich im Überschuß des Fällungsmittels; aus der Lösung scheiden sich beim Reiben mit einem Glasstab feine gelbe Kristalle aus. 
Bekanntlich findet sich Cholin in den Pflanzensamen in großer Verbreitung vor; auch Betain ist schon in manchen Samen gefunden worden.

Beide Basen fanden sich in den Sonnenblumensamen nur in kleiner Quantität vor.

4. Embryo des Weizenkorns (von Triticum vulgare).

Unter dem Namen "Weizenkeime» ist als Abfall des Müllereigewerbes im Handel ein Material zu erhalten,. welches in der Hauptsache aus den ungekeimten Embryonen des Weizens besteht, aber auch in gewisser Menge Teilchen des Endosperms einschließt. ${ }^{1}$ ) Dieses Material ist in unserem Laboratorium schon mehrfach untersucht worden $;^{2}$ ) es wurden daraus wiederholt Asparagin und Allantoin dargestellt, ${ }^{3}$ ) ferner auch Cholin und Betain. Um zu prüfen, ob sich darin auch Arginin vorfindet, extrahierten wir $1^{1 / 2}$ Kilo zuvor entfetteter "Weizenkeime" mit kaltem Wasser unter Zusatz von Chloroform als Antiseptikum. Das durch Filtration vom Ungelösten getrennte Extrakt wurde so verarbeitet, wie es oben für das Extrakt aus Lupinensamen angegeben worden ist. Dabei erhielten wir $0,64 \mathrm{~g}$ Argininnitrat. Allem Anschein nach war dieses Salz fast völlig rein. Das daraus dargestellte Argininkupfernitrat kristallisierte sofort in der charakteristischen Form; der Schmelzpunkt der Kristalle lag bei $113-114^{\circ}$. Bei der Zerlegung mittels Schwefelwasserstoff lieferte diese Kupferverbindung ein neutrales Nitrat, welches das Aussehen des Argininnitrats besaß und die Argininreaktionen gab.

Die im Filtrat vom Argininsilberniederschlag noch enthaltenen Basen fällten wir aus der zuvor vom Silber und vom Baryt befreiten Flüssigkeit durch Phosphorwolframsäure aus.

1) Daneben finden sich in kleiner Menge auch wohl noch andere Verunreinigungen vor; doch war ihre Quantität in dem von uns verwendeten Material äußerst gering.

$\left.{ }^{2}\right)$ Wir verweisen insbesondere auf die Abhandlung von S. Frank furt, Landwirtsch. Versuchsstationen, Bd. 47, S. 449.

s) Allantoin wurde in diesem Material zuerst von Richardson und Cramton (Berichte der d. chem. Gesellschaft, Bd. 19, S. 1180) gefunden. 
Den Niederschlag zerlegten wir durch Baryumhydroxyd und führten sodann die in Freiheit gesetzten Basen in die Chlorhydrate über, welche bald zu kristallisieren begannen. Der in Weingeist lösliche Teil des Chlorhydratgemenges bestand allem Anschein nach nur aus den salzsauren Salzen der in den Weizenkeimen schon nachgewiesenen Basen Gholin und Betain. Mit einer Reindarstellung des salzsauren Cholins haben wir uns nicht befaßt, da ein nochmaliger Nachweis dieser in den Pflanzensamen sehr verbreiteten Base als unnötig erschien; dagegen untersuchten wir das salzsaure Betain, nachdem dieses Salz durch Behandlung mit kaltem absoluten Alkohol und darauffolgendes Umkristallisieren aus Wasser vom salzsauren Cholin befreit worden war. Es gab die Betainreaktionen. Das daraus dargestellte Chloraurat kristallisierte aus Wasser in glänzenden Blättchen und Nadeln, deren Goldgehalt der Theorie $(43,1 \% \mathrm{Au})$ entsprach, wie folgende Angaben beweisen:

$0,2540 \mathrm{~g}$ bei $100^{\circ}$ getrocknete Substanz gaben $0,1090 \mathrm{~g}$ $=42,92 \% \mathrm{Au}$.

In den Weizenkeimen finden sich also in kleiner Menge zwei als Produkte des Eiweißumsatzes in den Pflanzen auftretende Stickstoffverbindungen, nämlich Asparagin und Arginin vor. Daneben treten Allantoin, über dessen Ursprung etwas Sicheres nicht auszusagen ist, sowie Cholin und Betain auf.

In den Weizenkeimen fallen nach der von S. Frankfurt ausgeführten Bestimmung 0,8\% Stickstoff (=12,4\% des Gesamtstickstoffs) auf nichtproteinartige Vorbindungen. Es ist fraglich, ob dieser Betrag vollständig durch die obengenannten aus den Weizenkeimen dargestellten Stickstoffverbindungen gedeckt wird.

5. Sogenannte Erdnußkeime (von Arachis hypogaea).

Da auf Grund der an den Weizenkeimen gemachten Beobachtungen vermutet werden konnte, daß diejenigen Teile der Samenkörner, aus denen bei der Keimung die ersten Blättchen und die Wurzeln sich bilden, relativ reich an nicht- 
proteinartigen Stickstoffverbindungen sind, so war es von Interesse, noch die sogenannten Erdnußkeime ${ }^{1}$ ) zu untersuchen. Wir erhielten solche durch die Gefälligkeit der Direktion der niederländischen Ölfabrik in Delft. Wie die Untersuchung unter dem Mikroskop zeigte, bestanden sie aus Embryonen, von denen der größte Teil der Kotyledonen und ein Teil des Blattkeims abgerissen war. Der Stickstoffgehalt dieses Materials wurde gleich 4,74\% der Trockensubstanz gefunden; auf nichtproteinartige Verbindungen fielen $0,42 \%$ Stickstoff, bestimmt nach Stutzers Methode..$\left.^{2}\right)$

Da diese Erdnußkeime ungefähr $45 \%$ Fett enthielten, so wurden sie zunächst grob zerkleinert und mit Hilfe von Äther vom größten Teil des Fetts befreit. Dás entfettete Material wurde sodann in Wasser, dessen Temperatur ca. $90^{\circ}$ betrug, unter Umrühren eingetragen, das so entstandene Extrakt sodann durch Abseihen vom Ungelösten getrennt. Dieses noch etwas trübe Extrakt behandelten wir sodann, so wie es oben für die Extrakte aus Lupinensamen angegeben ist, mit Tannin und mit Bleiessig.

Zur Darstellung von Arginin diente ein Extrakt aus ca. $2^{1} / 2 \mathrm{~kg}$ Erdnußkeimen. Die Art und Weise, in der dieses Extrakt verarbeitet wurde, entsprach ganz den weiter oben, im Abschnitt 1 gemachten Angaben. Wir erhielten 1,3 g fast völlig

1) Diese sog. Erdnußkeime werden auf mechanische Weise von den Erdnußsamen abgetrennt, ehe man die letzteren zur Darstellung von Öl verwendet.

2) Analytische Belege: $60 \mathrm{~g}$ Erdnußkeime wurden gröblich zerkleinert und sodann mit Äther behandelt; dabei erhielten wir eine ätherische Lösung, welche $24,7 \mathrm{~g}$ Fett einschloß, und einen nicht ganz fettfreien Rückstand, der in lufttrockenem Zustande $36 \mathrm{~g}$ wog. In diesem Rückstand bestimmten wir den Gesamtstickstoff und die auf nichtproteinartige Verbindungen fallende Stickstoffmenge nach $\mathrm{Kjeldahls} \mathrm{Methode.} \mathrm{a)} \mathrm{Ge} \mathrm{-}$ samtstickst off: $0,8950 \mathrm{~g}$ Trockensubstanz (=1,000 g lufttrocken) gaben $0,0764 \mathrm{~g}=8,53 \%$ Stickstoff. Eine zweite Bestimmung gab das gleiche Resultat. b) Stickstoff in nichtproteinartigen Verbindungen: 1. $0,8950 \mathrm{~g}$ Trockensubstanz ( $=1,000 \mathrm{~g}$ lufttrocken) gaben $0,00637 \mathrm{~g}=$ $0,71 \%$ Stickstoff. 2. $0,8950 \mathrm{~g}$ Trockensubstanz gaben $0,00665 \mathrm{~g}=0,74 \%$ Stickstoff. Aus diesen Daten berechnen sich für die ursprüngliche, fetthaltige Substanz die oben angegebenen Werte. 
reines Argininnitrat. Dieses Salz wurde in Argininkupfernitrat übergeführt. Letzteres kristallisierte in der charakteristischen Form. Der Schmelzpunkt der Kristalle lag zwischen 112 und 114 ${ }^{\circ}$. Die Bestimmung des Kupfergehalts in der durch Erhitzen auf $80^{\circ}$, dann auf $100^{\circ}$ vom Kristallwasser befreiten Substanz gab Zahlen, die dem von der Theorie geforderten Werte $(11,89 \% \mathrm{Cu})$ nahe liegen, wie folgende Angaben beweisen:

a) $0,3160 \mathrm{~g}$ Substanz gaben $0,460 \mathrm{~g} \mathrm{CuO}=11,63 \% \mathrm{Cu}$

b) 0,2380 , $, \quad 0,0340$, $=11,43 \%$,

Nach dem Auskristallisieren des Argininkupfernitrats blieb in kleiner Menge eine Mutterlauge übrig, die wir mittels Schwefelwasserstoff vom Kupfer befreiten und sodann im Wasserbade einengten; sie lieferte in kleiner Quantität Kristalle, die im Aussehen und in den Reaktionen mit Argininnitrat übereinstimmten. Es konnte also nicht nachgewiesen werden, daß in dem aus den Erdnußkeimen erhaltenen Argininnitrat noch ein anderes Nitrat enthalten war; auch fand sich darin nur eine Spur von Asche vor. Das bei Zerlegung des schön kristallisierten Argininkupfernitrats mit Schwefelwasserstoff erhaltene neutrale Nitrat gab die Argininreaktionen.

Vermutlich war das Arginin begleitet von etwas Histidin; doch war die Quantität der nach ihrem Verhalten gegen Fällungsmittel für Histidin zu erklärenden Substanz zu gering, um sie mit Sicherheit identifizieren zu können. Im Filtrat vom Argininsilberniederschlage suchten wir Lysin nachzuweisen; doch war das Resultat ein negatives. Dagegen fand sich in diesem Filtrat Cholin vor, dessen Vorhandensein in den Erdnußkeimen früher schon von E. Schulze ${ }^{1}$ ) nachgewiesen worden ist. Das salzsaure Salz dieser Base wurde aus dem in früher beschriebener Weise dargestellten Chlorhydratgemenge durch Ausziehen mit Alkohol gewonnen und sodann in die schwer lösliche Verbindung mit Mercurichlorid übergeführt. Letztere lieferte nach dem Umkristallisieren bei der Zerlegung durch Schwefelwasserstoff salzsaures Cholin.

Wir untersuchten ferner die stickstoffhaltigen Stoffe, welche aus einem in oben beschriebener Weise dargestellten

1) Landwirtschaftliche Versuchssationen, Bd. 44, S. 23. 
und gereinigten Extrakt aus Erdnußkeimen durch Mercurinitrat gefällt wurden. Die durch dieses Reagens im Filtrat vom Bleiessigniederschlag hervorgebrachte Fällung wurde abfiltriert, das Filtrat sodann mit noch etwas Mercurinitrat und, zur Abstumpfung der Säure, mit etwas Natriumkarbonnat versetzt. So erhielten wir zwei Fällungen, die getrennt untersucht wurden. Die erste Fällung, verarbeitet in der im Abschnitt 1 schon angegebenen Weise, lieferte Vernin. Letzteres schied sich aus der stark eingeengten Flüssigkeit zunächst in amorphem $\mathrm{Zu}-$ stande aus, wurde aber nach dem Auflösen in heißem Wasser kristallinisch. Noch einmal aus Wasser umkristallisiert und sodann im Exsikkator getrocknet, bildete es eine weiße atlasglänzende Substanz, deren Eigenschaften den im Abschnitt 1 für das Vernin gemachten Angaben entsprachen; bei der Spaltung durch verdünnte Salzsäure lieferte es Guanin, das wir mit Hilfe der oben angegebenen Reaktionen identifizierten.

Bei der Verarbeitung des zweiten Mercurinitratniederschlags wurde ein Sirup erhalten, aus welchem eine in kaltem Wasser sehr schwer lösliche Substanz in kleiner Quantität sich abschied. Sie löste sich in Ammoniakflüssigkeit; die Lösung lieferte beim Verdunsten einen im Aussehen mit Tyrosin übereinstimmenden Körper. Da dieses Produkt sowobl die von Hoffmann wie die von Piria und von Mörner angegebenen Tyrosinreaktionen gab, so ist nicht zu bezweifeln, daß wirklich Tyrosin vorlag. Dasselbe löste sich auch in verdünnter Salpetersäure; beim Erwärmen nahm die Lösung nur gelbe Färbung an.

In den Erdnußkeimen haben wir also Arginin, Tyrosin, Vernin und Cholin nachweisen können. Die Ausbeute an Arginin war sehr gering; sie betrug nur ca. 0,05\% der Trockensubstanz der Keime. Noch geringer war die Ausbeute an Vernin, indem wir aus zwei Kilo der lufttrocknen Keimé weniger als ein halbes Gramm dieses Produktes erhielten. Auch Tyrosin wurde nur in sehr kleiner Menge gewonnen. ${ }^{1}$ ) Etwas größer war die Ausbeute an Cholin.

1) $\mathrm{Da}$ aber die Ausfällung des Tyrosins durch Mercurinitrat eine ganz unvollständige ist, so muß es als möglich bezeichnet werden, daß diese Aminosäure sich in dem untersuchten Extrakt in etwas größerer Menge vorfand. 


\section{Rückblick auf die Resultate.}

Wie aus den im vorigen gemachten Mitteilungen zu ersehen ist, haben wir aus fünf Sorten ungekeimter Samen Arginin abscheiden können. Die größte Ausbeute $(0,3-0,4$ Teile aus 100 Teilen Samentrockensubstanz) erhielten wir bei Lupinus luteus, die niedrigste bei Helianthus annuus. Unsere Versuche lieferten einen neuen Beweis nicht nur für die Verbreitung des Arginins in den Pflanzen, sondern auch für den hohen Wert der von Kossel angegebenen Isolierungsmethode; denn mit Hilfe dieser Methode gelang es, die genannte Base auch aus Objekten darzustellen, die nur einige Hundertstel Prozent davon enthielten. Es bedarf aber noch einer Erörterung der Frage, ob etwa in denjenigen Fällen, in welchen wir nur sehr wenig Arginin erhielten, das letztere sich erst während der Verarbeiturig der Extrakte aus Proteinstoffen gebildet hat. Diese Frage muß wohl verneint werden. Die Extrakte wurden gleich nach ihrer Darstellung mit Hilfe von Tannin und Bleiessig von den Proteinstoffen so vollständig wie möglich befreit, dann bei ganz schwachsaurer Reaktion und bei einer weit unter $100 \mathrm{Grad}$ liegenden Temperatur eingeengt. Daß die letztere Operation die Zersetzung von etwa in der Flüssigkeit noch vorhandenen Resten von Proteinstoffen unter Argininbildung zur Folge hatte, ist höchst unwahrscheinlich. Ebenso wenig ist anzunehmen, daß dies bei einer der anderen von uns ausgeführten Operationen eingetreten ist. ${ }^{1}$ )

Von den Stickstoffverbindungen, die als Produkte des Eiweißumsatzes in den Pflanzen auftreten, sind in ungekeimten Pflanzensamen drei, nämlich Arginin, Tyrosin und As-

1) Wenn bei der Verarbeitung von Samen oder anderen eiweißreichen Pflanzenteilen nach dem von uns angegebenen Verfahren Arginin als Spaltungsprodukt sich bildete, so müßte man doch auch diese Base stets erhalten. Dies ist aber nicht der Fall; so erhielten wir z. B. ein negatives Resultat bei Erbsensamen. Aus Sonnenblumensamen haben wir, wie aus unsern Mitteilungen sich ersehen läßt, nur eine minimale Argininmenge, weniger als 0,005\%, darstellen können. Wenn die Ausbeute eine so geringe ist, so kann selbstverständlich die Frage, ob das Arginin erst durch Spaltung entstanden ist, nicht so sicher beantwortet werden, als bei größerer Ausbeute. 
paragin, nachgewiesen worden. Die Frage nach der Herkunft dieser Stoffe in den Samen läßt sich in zweifacher Weise beantworten. Ist die Annahme richtig, daß Aminosäuren und andere beim Eiweißumsatz auftretende Stickstoffverbindungen aus anderen Pflanzenteilen in die reifenden Samen einwandern und hier zur Eiweißsynthese verwendet werden, so können Reste solchen Materials in den reifen Samen sich noch vorfinden; ihre Quantität wird um so geringer sein, je besser die Samen ausgereift sind. Andererseits ist es auch denkbar, daß Stoffe solcher Art nach Vollendung der Samenreife in den Embryonen als Stoffwechselprodukte in geringer Quantität sich gebildet haben.

Aus zwei Samenarten konnten wir Vernin darstellen, eine Stickstoffverbindung, die aus ungekeimten Pflanzensamen bisher noch nicht dargestellt worden war. Es gelang uns, einige neue Reaktionen des Vernins aufzufinden, die nicht nur zum Nachweis dieses Körpers dienen können, sondern auch über die Beschaffenheit der in ihm mit dem Guanin verbundenen Atomgruppe Aufschluß geben; sie führen zu der Schlußfolgerung, daß das Vernin eine Kohlenhydratgruppe einschließt und als ein Glukosid zu betrachten ist.

Die in ungekeimten Pflanzensamen bis jetzt nachgewiesenen nichtproteinartigen Stickstoffverbindungen finden sich darin nur in so kleiner Quantität vor, daß durch sie die im ganzen auf solche Verbindungen fallende Stickstoffmenge, bestimmt nach Stutzers Verfahren, allem Anschein nach nur zum Teil gedeckt wird. Als nicht unwahrscheinlich kann es bezeichnet werden, daß in ungekeimten Pflanzensamen auch peptonartige Stoffe sich finden; doch sind neue Untersuchungen erforderlich, um darüber etwas Bestimmtes aussagen zu können. 